\title{
TOWARDS PRECISION DETERMINATION OF UNINTEGRATED PARTON DENSITY FUNCTIONS
}

\author{
H. JUNG \\ $D E S Y, F R G$ \\ E-mail: hannes.jung@desy.de \\ A.V. KOTIKOV \\ BLTHPH, JINR, Dubna, Russia \\ E-mail:kotikov@theor.jinr.ru \\ A.V. LIPATOV and N.P. ZOTOV \\ SINP, Lomonosov Moscow State University, Russia \\ E-mail: zotov@theory.sinp.msu.ru, lipatov@theory.sinp.msu.ru
}

\begin{abstract}
First attempts are described to determine the unintegrated Parton Density Function of the gluon from a fit to measurements of the structure function $F_{2}\left(x, Q^{2}\right)$ and also $F_{2}^{c}\left(x, Q^{2}\right)$ measured at HERA. Reasonable descriptions of both structure functions are obtained, but the gluon densities determined are different.
\end{abstract}

Keywords: pQCD; unintegrated parton densities; $k_{\perp}$-factorization.

\section{Introduction}

Unintegrated parton density functions (uPDFs) are best suited to study details of the hadronic final state in high energy $e p$ and also in $p p$ collisions (for a review see $[1-7])$. In general, the production cross section for jets, heavy quarks or gauge bosons can be written as a convolution of the uPDF $\mathcal{A}\left(x, k_{\perp}^{2}, \bar{q}\right)$ with the partonic off-shell cross section $\hat{\sigma}\left(x_{i}, k_{\perp}^{2}\right)$, with $x_{i}, k_{\perp}$ being the longitudinal momentum fraction and the transverse momentum of the interacting parton $i$ and $\bar{q}$ being the factorization scale. For example the cross section for $e p \rightarrow$ jets $+X$ can be written as:

$$
\begin{aligned}
\frac{d \sigma^{j e t s}}{d E_{T} d \eta}= & \sum_{i} \iiint d x_{i} d Q^{2} d \ldots \\
& {\left[d k_{\perp}^{2} x_{i} \mathcal{A}\left(x_{i}, k_{\perp}^{2}, \bar{q}\right)\right] \hat{\sigma}\left(x_{i}, k_{\perp}^{2}\right) }
\end{aligned}
$$

At high energies, the gluon density is dominating for many processes, therefore here only the gluon uPDF is considered. It has already been shown in [8], that the predictions of the total cross section as well as differential distributions for heavy quark production at HERA and the LHC agree well in general with those coming from fixed NLO calculations. However, the details depend crucially on a precise knowledge of the UPDF. Therefore precision fits to inclusive and exclusive measurements have to be performed to determine precisely the free parameters of the uPDF: the starting distribution function at a low scale $\bar{q}_{0} \sim 1 \mathrm{GeV}$ as well as parameters connected with $\alpha_{\mathrm{s}}$ and details of the splitting functions for the perturbative evolution.

The previously available uPDFs (a overview is given in [3-5]) were only adjusted to describe $F_{2}$, but no real fit of the parameters of the starting distribution was performed, neither the experimental uncertainties were treated as is done in global QCD analyses in the collinear approach (for example from CTEQor MRST).

Here attempts to determine the uPDF of the gluon from pQCD fits using the CCFM 
evolution equation [9]. to the inclusive structure function $F_{2}\left(x, Q^{2}\right)$ as well as $F_{2}^{c}\left(x, Q^{2}\right)$, as measured by the HERA experiments, are described.

\section{The method}

The unintegrated gluon density is determined by a convolution of the nonperturbative starting distribution $\mathcal{A}_{0}(x)$ and the CCFM evolution denoted by $\tilde{\mathcal{A}}\left(x, k_{\perp}, \bar{q}\right)$ :

$$
\begin{aligned}
x \mathcal{A}\left(x, k_{\perp}, \bar{q}\right)= & \int d x^{\prime} \mathcal{A}_{0}\left(x^{\prime}\right) \cdot \frac{x}{x^{\prime}} \\
& \tilde{\mathcal{A}}\left(\frac{x}{x^{\prime}}, k_{\perp}, \bar{q}\right)
\end{aligned}
$$

In the perturbative evolution the gluon splitting function $P_{g g}$ including non-singular terms (as described in detail in [10]) is applied.

The distribution $\mathcal{A}_{0}$ is parameterized at the starting scale $\bar{q}_{0}$ by:

$$
x \mathcal{A}_{0}(x)=N x^{-B_{g}} \cdot(1-x)^{C_{g}}\left(1-D_{g} x\right)
$$

The parameters $N_{g}, B_{g}, C_{g}, D_{g}$ of $\mathcal{A}_{0}$ are determined in the fit, which is based on the MINUIT package [11] and the extension by [12], such to minimize the $\chi^{2}$ defined by:

$$
\chi^{2}=\sum_{i}\left(\frac{(T-D)^{2}}{\sigma_{i}^{2}{ }^{\text {stat }}+\sigma_{i}^{2} \text { uncor }}\right)
$$

with $T$ being the theory value and $D$ the measurement with the corresponding statistical and uncorrelated systematic uncertainty.

\section{The structure function $F_{2}\left(x, Q^{2}\right)$}

The measurement of $F_{2}\left(x, Q^{2}\right)$ [13] is used in the range $x<0.005$ and $Q^{2}>5 \mathrm{GeV}^{2}$ to determine the uPDF. The parameters of $\mathcal{A}_{0}$ were investigated separately, and it was confirmed, that the data do not constrain the parameters $C_{g}$ and $D_{g}$, which were therefore fixed to $C_{g}=4$ and $D_{g}=0$. The starting distribution $\mathcal{A}_{0}$ was parameterized at $\bar{q}_{0}=1.2 \mathrm{GeV}$. The running coupling $\alpha_{\mathrm{s}}(\mu)$ was used in the 1-loop approximation in the region $\mu>\bar{q}_{0}$, and was kept fixed at $\alpha_{\mathrm{s}}\left(\bar{q}_{0}\right)$ for $\mu<\bar{q}_{0}$. A acceptable fit to the measured $F_{2}$ values was obtained with $\chi^{2} / n d f=111.8 / 61=1.83$ using only statistical and uncorrelated systematical uncertainties (compare to $\chi^{2} / n d f \sim 1.5$ in the collinear approach at NLO). In Fig. 1 the measurement is compared to the prediction of the structure function $F_{2}\left(x, Q^{2}\right)$ as obtained from the fit. Since in the cross section

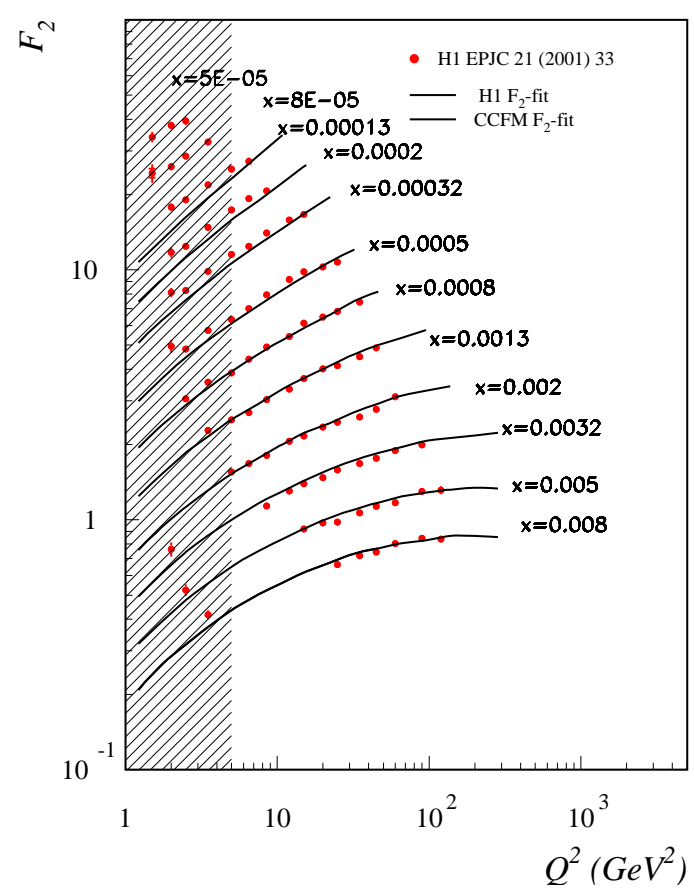

Fig. 1. The structure function $F_{2}\left(x, Q^{2}\right)$ as a function of $Q^{2}$ for different values of $x$. The data points are from [13]. The shaded area shows the region which is not used in the fit.

a product of the gluon density and $\alpha_{\mathrm{s}}$ enters (as well as $\alpha_{\mathrm{s}}$ enters in the pQCD evolution) also the dependence on the choice of $\Lambda_{q c d}$ was investigated (Fig. 2). A clear preference for $\Lambda_{q c d}^{(4)} \sim 0.13$ is observed, which corresponds to $\alpha_{\mathrm{s}}\left(m_{Z_{0}}\right)=0.118$ in the 1-loop approximation. 


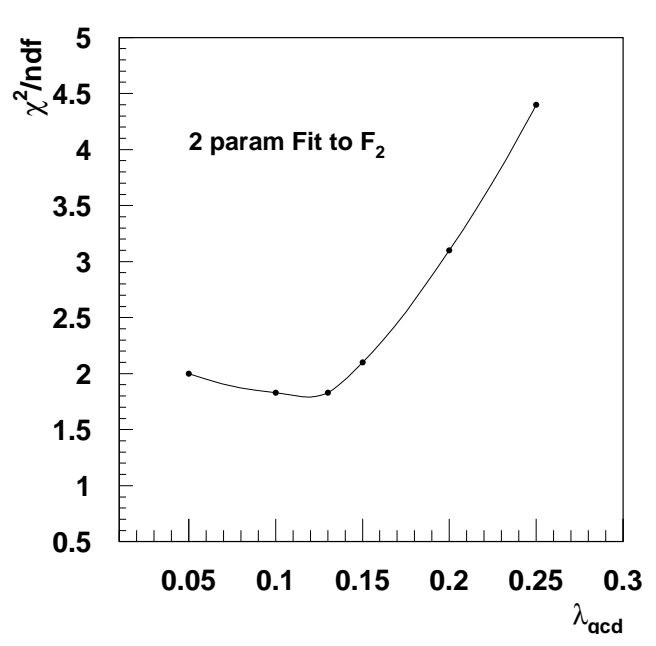

Fig. 2. $\chi^{2} / n d f$ dependence of the $F_{2}$ fit on the choice of $\Lambda_{q c d}$

\section{The structure function $F_{2}^{c}\left(x, Q^{2}\right)$ and the uPDF}

The structure function $F_{2}^{c}\left(x, Q^{2}\right)$ is directly sensitive to the gluon density. The measurements of [14] were used to determine the $\mathrm{uPDF}$ in the range $Q^{2} \geq 1.5 \mathrm{GeV}^{2}$. A acceptable fit to the measured $F_{2}^{c}$ values was obtained with $\chi^{2} / n d f=18.8 / 20=0.94$ using statistical and systematic uncertainties. In Fig. 3 the measurement is compared to the prediction of the structure function $F_{2}^{c}\left(x, Q^{2}\right)$ as obtained from the fit.

Since the fits to $F_{2}\left(x, Q^{2}\right)$ and $F_{2}^{c}\left(x, Q^{2}\right)$ were performed independently, it is interesting to compare the resulting unintegrated gluon distributions (Fig. 4). The small $x$ behavior of the gluon distribution is very different. It has been explictly checked, that the measured $F_{2}\left(x, Q^{2}\right)$ cannot be described with the uPDF obtained from the fit to $F_{2}^{c}\left(x, Q^{2}\right)$ and vice versa.

\section{The structure function $F_{L}^{c}\left(x, Q^{2}\right)$}

Using the different $\mathrm{uPDFs}$ described in the previous sections we calculate the longitu- dinal structure function $F_{L}\left(x, Q^{2}\right)$ at fixed $W$ in the framework of [16]. The predictions are compared with the measurements at fixed $W$ of $\mathrm{H} 1$ [15] in Fig. 5. The general trend is nicely described, however the predicted shape of $F_{L}$ at medium $Q^{2}$ is different and precise measurements would be very welcome.

\section{Acknowledgments}

Many thanks go the E. Perez for the support with the fit program and the experimental error treatment. Thanks also to the organizers of this very interesting conference.

\section{References}

1. H. Jung, Phys. Rev. D 65, 034015 (2002). DESY-01-136, hep-ph/0110034

2. H. Jung, Mod. Phys. Lett. A19, 1 (2004). hep-ph/0311249

3. Small X Collaboration; B. Andersson et

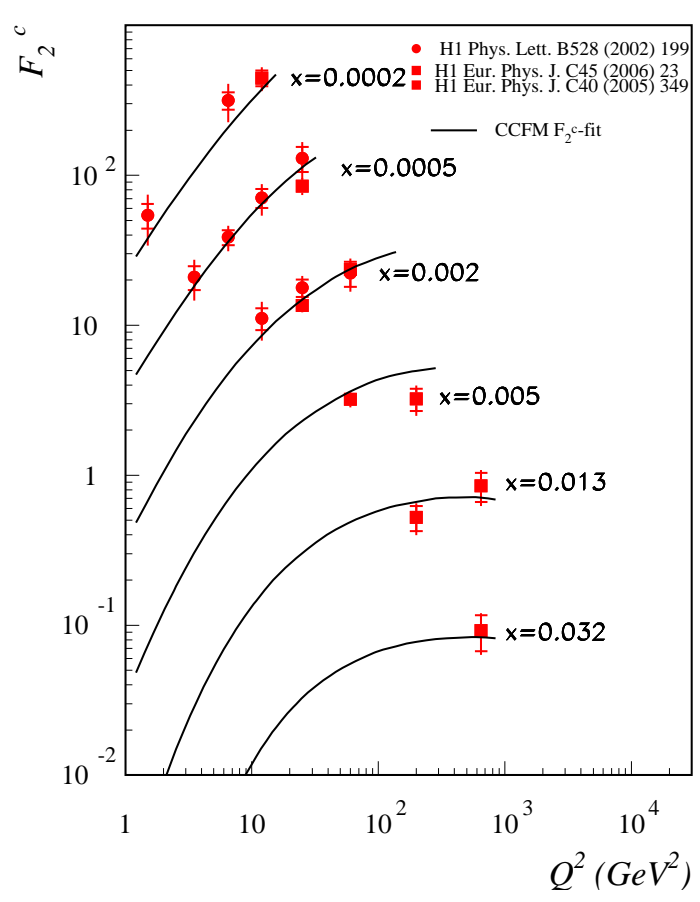

Fig. 3. The structure function $F_{2}^{c}\left(x, Q^{2}\right)$ as a function of $Q^{2}$ for different values of $x$. The data points are from [14]. 


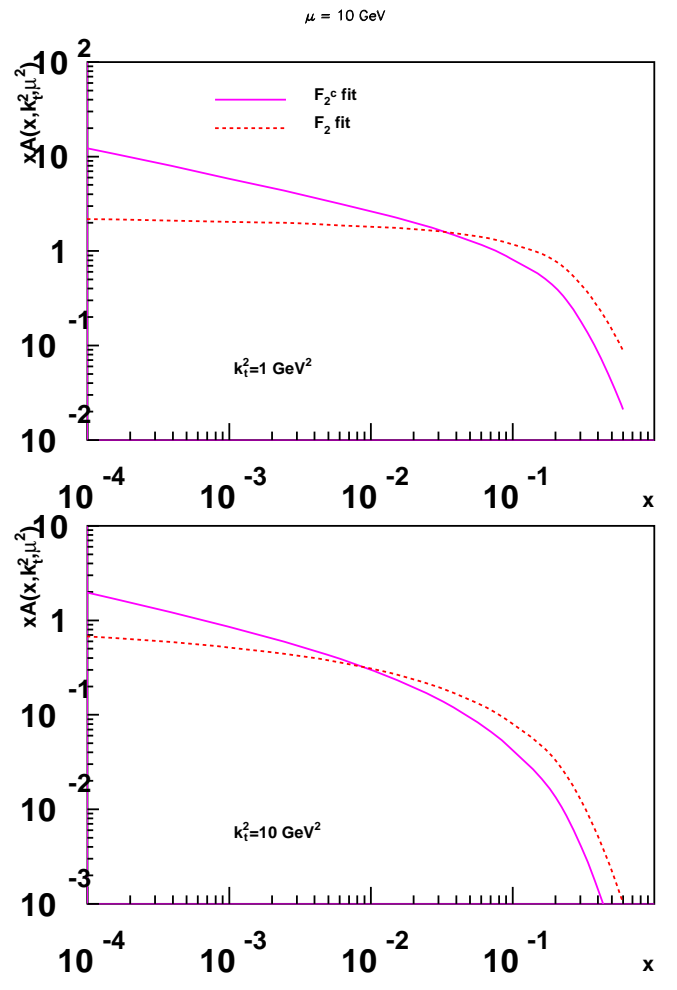

Fig. 4. The unintegrated gluon density as obtained from the fits to $F_{2}\left(x, Q^{2}\right)$ and $F_{2}^{c}\left(x, Q^{2}\right)$ as a function of $x$ for different values of $k_{\perp}$. The gluon density is evolved to a scale $\bar{q}=10 \mathrm{GeV}$.

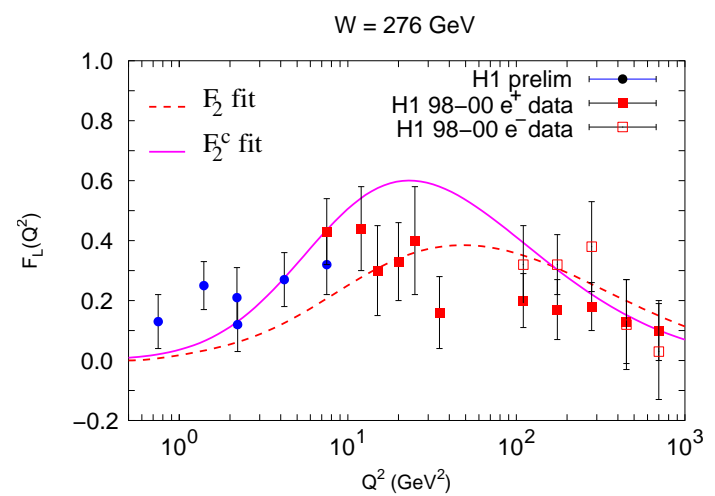

Fig. 5. The longitudinal structure function $F_{L}\left(x, Q^{2}\right)$ at fixed $W$ as measured by [15]. The solid curve is solid curve is the prediction using the fit to $F_{2}$ whereas the dashed curve uses the uPDF obtained from a fit to $F_{2}^{c}$.

al., Eur. Phys. J. C 25, 77 (2002). hep-ph/0204115.
4. Small X Collaboration, J. R. Andersen et al., Eur. Phys. J. C35, 67 (2004). hep-ph/0312333.

5. Small X Collaboration, J. R. Andersen et al. (2006). hep-ph/0604189

6. H. Jung. DIS 2004, Strbské Pleso, Slovakia, hep-ph/0411287

7. J. Collins and H. Jung, Need for fully unintegrated parton densities, 2005. hep-ph/0508280.

8. S. Alekhin et al., Hera and the $L H C-a$ workshop on the implications of HERA for LHC physics: Proceedings Part $A$ and $B$, 2005. hep-ph/0601012, hep-ph/0601013 J. Baines et al. (2006). hep-ph/0601164.

9. M. Ciafaloni, Nucl. Phys. B 296, 49 (1988); S. Catani, F. Fiorani, and G. Marchesini, Phys. Lett. B 234, 339 (1990);

S. Catani, F. Fiorani, and G. Marchesini, Nucl. Phys. B 336, 18 (1990);

G. Marchesini, Nucl. Phys. B 445, 49 (1995).

10. H. Jung, Acta Phys. Polon. B33, 2995 (2002). hep-ph/0207239,

M. Hansson and H. Jung. DIS 2003, St. Petersburg, Russia, hep-ph/0309009

11. F. James and M. Roos, Comput. Phys. Commun. 10, 343 (1975).

12. J. Pumplin, D. R. Stump, and W. K. Tung, Phys. Rev. D65, 014011 (2002). hep-ph/0008191.

13. H1 Collaboration, C. Adloff et al., Eur. Phys. J. C21, 33 (2001). hep-ex/0012053.

14. H1 Collaboration, C. Adloff et al., Phys. Lett. B528, 199 (2002). hep-ex/0108039.

H1 Collaboration, A. Aktas et al., Eur. Phys. J. C45, 23 (2006). hep-ex/0507081; H1 Collaboration, A. Aktas et al., Eur. Phys. J. C40, 349 (2005). hep-ex/0411046

15. P. Newman, Int. J. Mod. Phys. A19, 1061 (2004). hep-ex/0312018 E. M. Lobodzinska (2003). hep-ph/0311180.

16. A. V. Kotikov, A. V. Lipatov, and N. P. Zotov, J. Exp. Theor. Phys. 101, 811 (2005). hep-ph/0403135;

A. V. Kotikov, A. V. Lipatov, and N. P. Zotov (2005). hep-ph/0503275. 